Journal of Animal and Veterinary Advances 11 (3): 426-430, 2012

ISSN: $1680-5593$

(C) Medwell Journals, 2012

\title{
Cloning of Structural Protein VPI Gene of Foot and Mouth Disease Virus and its Expression in Escherichia coli
}

\author{
${ }^{1 .}{ }^{2}$ Qiumei Shi, ${ }^{2}$ Guisheng Gao, ${ }^{2}$ Yanying Zhang, ${ }^{3}$ Hua Xiang, ${ }^{1}$ Zengqiang Yuan and ${ }^{4}$ Hongxuan He \\ ${ }^{1}$ Institute of Biophysics, Chinese Academy of Sciences, \\ State Key Laboratory of Brain and Cognitive Science, Chaoyang District, Beijing, China \\ ${ }^{2}$ Hebei Normal University of Science and Technology College of Animal Science, \\ Changli, Hebei, China \\ ${ }^{3}$ Veterinary Research Institute, Guangdong Provincial Academy of Agricultural Sciences, \\ Guangzhou, Guangdong, China \\ ${ }^{4}$ Institute of Zoology Chinese Academy of Science, Chaoyang District, Beijing, China
}

\begin{abstract}
In this study, the viral RNA was extracted from swine FMDV and then the fragment of VP1 was amplified with a primer pair by RT-PCR. The interest fragmen was inserted into pGEM-Teasy vector. There combinant plasmid was identified by restriction analysis and PCR. It was proved by DNA sequencing that the acquired recombinant contains complete VP1 gene. The homologie of the nucleotide sequence of VP1 gene were 95.9 and $96.2 \%$, respectively comparing with that of strai $\mathrm{O} / \mathrm{JPN} / 00$ and O-Tibet- 99 . Afterwards, the complete $V P 1$ gene from the identified recombin an was amplifie with another primer pair containing BamHI and XhoI sites by PCR and digested it with BamHI and XhoI. The expression vector $\mathrm{pET} 28 \mathrm{a}$ were digested by BamHI and XhoI, respectively. The target gene $V P 1$ was subcloned into vector pET28a. Positive clones named as pET28a-VP1 with interest gene were identificated by restriction analysis, PCR and DNA sequencing. Then there combinant was transformed into Escherichia coli BL21 (DE3) for VP1 expression. The interest gene was induced to express in E. coli with IPTG. The bacteria containing pET28a-VP1 were collected at different time and subsequently were examined by SDS-PAGE and Western-blotting.
\end{abstract}

Key words: FMDV, structrural protein, VP1 gene, clone, sequence analysis expression, China

\section{INTRODUCTION}

Foot and mouth disease was acute, febricity and highly contagious infectious diseases causing by foot and mouth disease virus (Francisco et al., 2001). Structrural protein VP1 function zone contained the main epitope of foot and mouth disease virus which could induce infection animal producing neutralizing antibody. It exposed in the virus particle surface, mutated easily and was paid more attention to the research (Logan et al., 1993). In the studying of recombinant vaccines, Kleid (1981) firstly applied recombinant DNA technology making VP1 gene expression in Escherichia coli. Expression product confirmed that it could induce cattle and pig neutralizing antibody response. Later, similar studys at home and abroad were performed (Ward et al., 1997; Mateu, 1995). Based on the important significance of VP1 genes to FMDV. Structrural protein VP1 genetic fragments was amplified from FMDV structrural genome then was cloned, expressed and identified in this test. It provided theoretical material for whole structrural protein P1 of FMDV expression and expression product as a diagnostic antigens.

\section{MATERIALS AND METHODS}

Cells and virus: Vero cells, FMDV O-DG-2005 strains, preserved with grinding coolant of neonatal rat carcass.

Primer design and synthesis: Primer including $V P 1$ gene: P1:5'-GTGGCCGAGGCGTGCCC-3', P2:5'GACATGTCCTCCTGCATCTG-3'. Product with 1286 bp was amplified which took $\mathrm{P} 1$ and $\mathrm{P} 2$ as primer. Primer with restriction enzyme sites of BamHI and Xhol. Restriction enzyme sites BamHI and XhoI were inserted into upstream and downstream primer: L1'-GCGGATCCACCACCTCTACAGGTGAG-3', L2 5'-GCCTCGAGTCAAAAGCTGTTTCACAG-3', Product with 656 bp was amplified which took L1 andL 2 as primer.

Corresponding Author: Zengqiang Yuan, Institute of Biophysics, Chinese Academy of Sciences, 
RT-PCR, cloning, recombinant plasmid identification and $V P 1$ gene sequenced: Grinding coolant of neonatal rat carcass was centrifuged and got Supernatant fluid. FMDV RNA was extracted from Supernatant fluid by the way of kit and cDNA was got by reverse transcription.

Then researchers took $\mathrm{cDNA}$ as templates $\mathrm{P} 1$ and $\mathrm{P} 2$ as primer and amplified by PCR. PCR product was subcloned into vector $\mathrm{pMD} 18-\mathrm{T}$ vector. Recombinant plasmid was identified by PCR and restriction enzyme. Positive clone (named pMD-VP1) was selected to sequence. Comparing this test strain sequence with home and abroad reference strains (19) sequence.

Construction of recombinant expression vector: Amplification fragments of $656 \mathrm{bp}$ was amplified by PCR with taking L1 and L2 as primers, recombinant plasmid pMD-VP1 as template. Meanwhile linear expression vector was got by double restriction enzyme the expression vector. Recombinant expression vector pET-VP1 was constructed by T4 DNA connection enzymes.

Identification of recombinant expression: Plasmid connecting product was transformed into $\mathrm{BL}_{21}\left(\mathrm{DE}_{3}\right)$ cell by routine method then single bacterial colony was took and cultivated. Plasmid was extracted and identificated by restriction analysis, PCR and DNA sequencing.

Inducted expression of recombinant plasmid: Positive recombinant strains was cultivated by routine method inducted and expressed. No inducing bacterium fluid, $\mathrm{BL}_{21}$ empty vector were set as contrast.

\section{Identification of expression product}

SDS-PAGE identification: Observing expression situation of positive recombinant strains by routine method.

Western-blotting identification: Protein which were separated by SDS-PAGE electrophoresis and mixed to swine FMDV positive serum then warmed for $1.5 \mathrm{~h}$. After washing, rabbit-anti-swine antibody marked by Horseradish Peroxidase (HRP) was warm for $1 \mathrm{~h}$ then washed, colour-displayed by adding Diaminobenzidine (DBA) and hydrogen peroxide. Taking cracking liquid which was not induced and induction content which was transformed by empty vector as controls.

\section{RESULTS AND DISCUSSION}

RT-PCR amplification of virus genome: CDNA templates were obtained by reverse transcription which taking genome of FMDV O-DG-2005 as templates, downstream primer as primer. Then $V P 1$ gene which was 1286 bp was

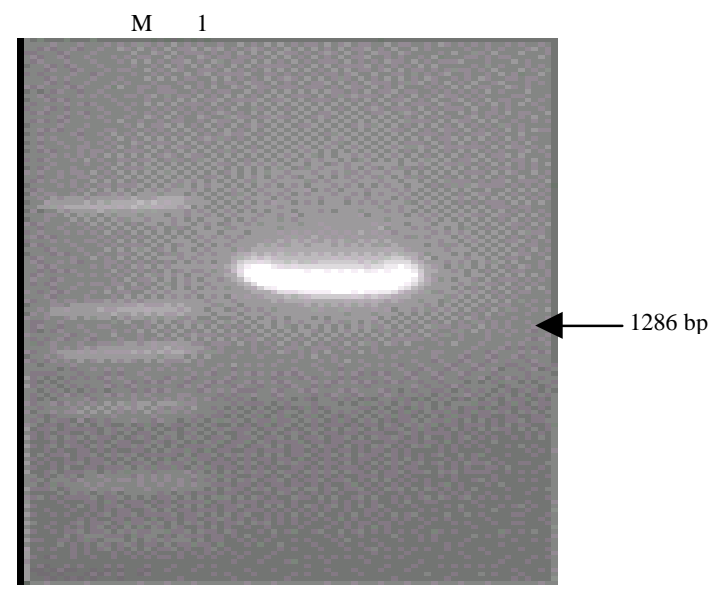

Fig. 1: VP1 of amplified by RT-PCR; M: 2000 bp DNA Marker; 1: VP1 gene

obtained by PCR and the size was consistent with expectation (Fig. 1). VP1 was subcloned into vector pMD18-T, transformed, identified then sequenced, confirming open reading frame of VP1 was correct in vector $\mathrm{pMD18-T}$. VP1 gene of FMDV of $\mathrm{O}$ type were compared with other reference strain by DNA Star software. The homologie of the nucleotide sequence of $V P 1$ gene was $>80 \%$, comparing with other $O$ type FMDV reference strain. Among those, the homologie with O-Tibet-99, O-JPN-00 were highest, reaching, respectively 96.2 and $95.9 \%$. The difference with O-ISA-8-83, O-ISA-74 and O-JAV-5-72 were the largest, reaching $39.7 \%$ (Table 1).

PCR amplification, transformation and identification of $V P 1$ gene: Taking positive plasmid of $\mathrm{pMD}$ VP1 of P1-P2 as templates, L1, L2 as primers, amplified VP1 gene and obtained purpose bands with the size of $656 \mathrm{bp}$ (Fig. 2). The PCR product was purified, double restriction analysis with BamHI and XhoI (Fig. 3) and recycled. Plasmid pET28a was double restriction analysis (about 5369 bp) (Fig. 4), recycled, linked, Transformed. After identification by PCR, researchers obtained purpose bands with the size of $656 \mathrm{bp}$ (Fig. 4-5). Recombinant plasmid pET-VP1 was single restriction analysis with XhoI and obtained a band with the size of 6025 bp (Fig. 2-5). After restriction analysis with XhoI and BamHI, researchers obtained purpose gene bands (656 bp) and vector (5369 bp-130) (Fig. 5) which was consistent with expection.

Induction expression and identification of pET-VP1: Recombinant plasmid $\mathrm{BL}_{21}\left(\mathrm{DE}_{3}\right)$ was transformed by $1 \mathrm{mmol} \mathrm{L}{ }^{-1} \mathrm{IPTG}$ inducing expression for $4 \mathrm{~h}$. Expression host bacterium was examined by SDS-PAGE then dyeinged. In the place of relative molecular mass 28,000 
Table 1: Homologic comparasion of the nucleotide sequences of VPI gene between this and other reference FMDV strains

\begin{tabular}{|c|c|c|c|c|c|c|c|c|c|c|c|c|c|c|c|c|c|c|c|c|c|c|}
\hline \multirow[b]{2}{*}{ Divergence } & \multicolumn{22}{|c|}{ Identity (\%) } \\
\hline & No. & 1 & 2 & 3 & 4 & 5 & 6 & 7 & 8 & 9 & 10 & 11 & 12 & 13 & 14 & 15 & 16 & 17 & 18 & 19 & 20 & No. \\
\hline O-VIT-7-97 & 1 & - & 18.8 & 94.8 & 79.5 & 80.6 & 81.2 & 85.6 & 84.7 & 81.4 & 80.4 & 85.3 & 79.8 & 85.6 & 81.7 & 85.3 & 80.9 & 39.3 & 38.8 & 38.8 & 83.7 & 1 \\
\hline O-Akesu-CHA-58 & 2 & 21.2 & - & 81.8 & 80.8 & 81.7 & 86.2 & 83.3 & 82.9 & 80.0 & 83.3 & 82.9 & 83.1 & 3.3 & 80.6 & 2.8 & 5.6 & 40.2 & 40.5 & 40.5 & 2.6 & 2 \\
\hline O-CAM-2-98 & 3 & 5.3 & 20.9 & - & 80.6 & 81.4 & 80.6 & 85.0 & 84.4 & 81.8 & 79.7 & 84.7 & 79.8 & 85.0 & 81.1 & 84.7 & 80.0 & 40.2 & 39.3 & 39.3 & 83.1 & 3 \\
\hline $\mathrm{O}-\mathrm{CT}$ & 4 & 24.7 & 22.7 & 22.8 & - & 90.6 & 79.7 & 82.0 & 81.7 & 81.7 & 78.4 & 82.2 & 78.1 & 82.5 & 83.6 & 82.0 & 79.5 & 40.4 & 41.9 & 42.3 & 81.5 & 4 \\
\hline O-GHA-5-93 & 5 & 23.1 & 21.4 & 21.7 & 10.3 & - & 77.9 & 83.9 & 82.9 & 81.8 & 76.7 & 83.3 & 77.0 & 83.7 & 83.3 & 83.1 & 77.6 & 38.5 & 41.0 & 41.3 & 82.2 & 5 \\
\hline $\mathrm{O}-\mathrm{HKN}$ & 6 & 22.1 & 15.6 & 22.7 & 24.2 & 26.8 & - & 82.3 & 82.2 & 77.5 & 87.6 & 81.8 & 87.8 & 82.5 & 78.9 & 81.8 & 96.9 & 39.0 & 39.7 & 40.1 & 82.0 & 6 \\
\hline O-IR & 7 & 16.5 & 19.4 & 17.1 & 21.2 & 18.6 & 20.6 & - & 96.9 & 81.7 & 81.4 & 97.2 & 82.2 & 97.8 & 82.6 & 96.9 & 81.8 & 39.4 & 40.1 & 40.1 & 94.1 & 7 \\
\hline O-JPN-00 & 8 & 17.7 & 19.8 & 17.9 & 21.7 & 19.9 & 20.8 & 3.2 & - & 80.8 & 81.2 & 99.1 & 81.7 & 99.1 & 81.2 & 98.7 & 81.7 & 39.4 & 40.2 & 40.2 & 95.9 & 8 \\
\hline $\mathrm{O}-\mathrm{K}$ & 9 & 18.9 & 20.7 & 18.0 & 18.6 & 18.4 & 24.3 & 18.5 & 19.9 & - & 75.3 & 81.4 & 76.1 & 81.7 & 86.7 & 81.4 & 76.7 & 41.3 & 41.0 & 41.3 & 80.4 & 9 \\
\hline $\mathrm{O}-\mathrm{PI}$ & 10 & 23.0 & 19.5 & 23.9 & 26.0 & 28.7 & 13.8 & 21.8 & 22.0 & 27.5 & . & 81.1 & 95.6 & 81.5 & 77.6 & 81.1 & 86.2 & 38.5 & 38.7 & 39.0 & 80.3 & 10 \\
\hline $\mathrm{O}-\mathrm{SK}$ & 11 & 16.9 & 19.8 & 17.5 & 21.0 & 19.5 & 21.3 & 2.9 & 0.9 & 19.0 & 22.2 & - & 81.4 & 99.4 & 81.5 & 99.7 & 81.4 & 39.7 & 40.5 & 40.5 & 95.6 & 11 \\
\hline O-TAW-81-97 & 12 & 23.6 & 19.3 & 23.3 & 26.0 & 27.8 & 13.3 & 20.3 & 21.0 & 25.9 & 4.2 & 21.4 & - & 82.0 & 77.0 & 81.4 & 86.2 & 38.2 & 38.0 & 38.5 & 80.8 & 12 \\
\hline O-Tibet-99 & 13 & 16.5 & 19.4 & 17.1 & 20.5 & 18.8 & 20.4 & 2.2 & 0.9 & 18.5 & 21.6 & 0.6 & 20.5 & - & 82.2 & 99.1 & 82.0 & 39.4 & 40.7 & 40.7 & 96.2 & 13 \\
\hline O-UGA-5-96 & 14 & 21.3 & 22.8 & 22.0 & 18.9 & 19.4 & 25.2 & 20.2 & 22.2 & 12.0 & 26.9 & 21.7 & 27.5 & 20.8 & - & 81.5 & 78.7 & 41.9 & 41.9 & 42.6 & 80.9 & 14 \\
\hline O-UKG-12-01 & 15 & 16.9 & 20.0 & 17.5 & 21.2 & 19.7 & 21.3 & 3.2 & 1.3 & 19.0 & 22.2 & 0.3 & 21.4 & 0.9 & 21.7 & - & 81.4 & 40.1 & 40.8 & 40.8 & 95.3 & 15 \\
\hline $\mathrm{O}-\mathrm{C}$ & 16 & 22.6 & 16.4 & 23.7 & 24.4 & 27.3 & 3.2 & 21.3 & 21.5 & 25.5 & 15.6 & 22.0 & 15.3 & 21.1 & 25.4 & 21.9 & - & 39.1 & 39.7 & 40.1 & 81.5 & 16 \\
\hline O-ISA-8-83 & 17 & 124.5 & 119.7 & 119.3 & 119.0 & 128.8 & 126.2 & 124.0 & 124.0 & 109.4 & 129.0 & 122.2 & 130.1 & 124.0 & 111.6 & 120.6 & 125.4 & - & 80.9 & 81.5 & 39.7 & 17 \\
\hline O-ISA-1-74 & 18 & 127.9 & 118.5 & 124.9 & 111.6 & 116.4 & 122.1 & 122.0 & 121.1 & 111.0 & 128.6 & 119.3 & 131.5 & 118.4 & 111.8 & 117.7 & 122.1 & 22.7 & - & 98.3 & 39.7 & 18 \\
\hline O-JAI & 19 & 127.5 & 118.3 & 122.8 & 110.2 & 114.8 & & 121.5 & 120.6 & 109.4 & 126.6 & 118.9 & 128.6 & 3.9 & 108.9 & 117.4 & 120.4 & 21.7 & 1.7 & - & 39.7 & 19 \\
\hline \multirow[t]{2}{*}{ O-DG-05 } & 20 & 19.0 & 20.3 & 19.7 & 21.9 & 21.0 & 21.0 & 6.3 & 4.2 & 20.3 & 23.3 & 4.6 & 22.3 & 3.9 & 22.6 & 4.9 & 21.7 & 122.3 & 124.0 & 123.5 & - & 20 \\
\hline & 1 & 2 & 3 & 4 & 5 & 6 & 7 & 8 & 9 & 10 & 11 & 12 & 13 & 13 & 14 & 15 & 16 & 17 & 18 & 19 & 20 & \\
\hline
\end{tabular}

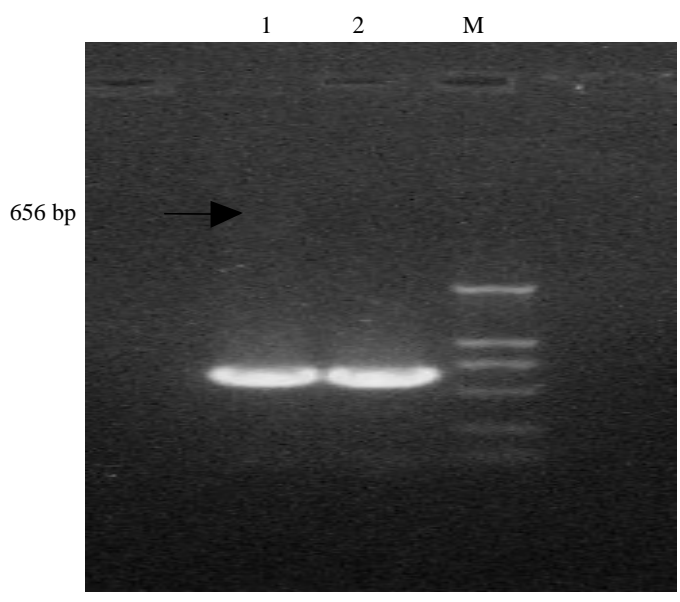

Fig. 2: VP1 amplified by PCR 1.2. VP1 gene (656 bp), M: DL 2000 bp DNA Marker

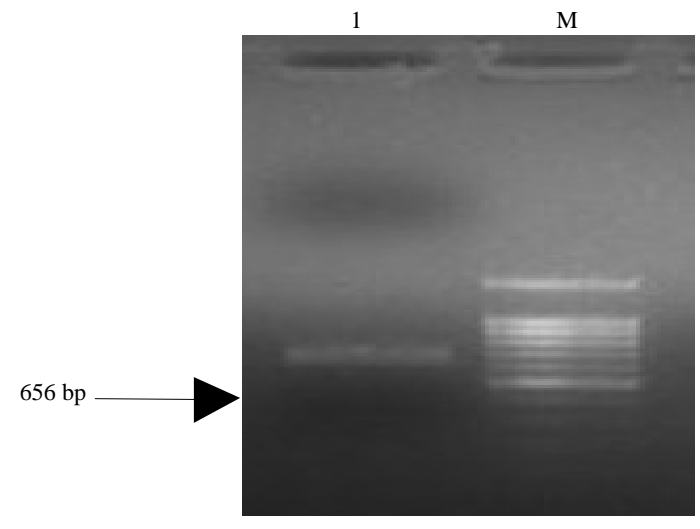

Fig. 3: Identification of Double digested of VP1. 1: Double digested products of VP1 with BamHI and XhoI; M: 100 bp DNA Marker

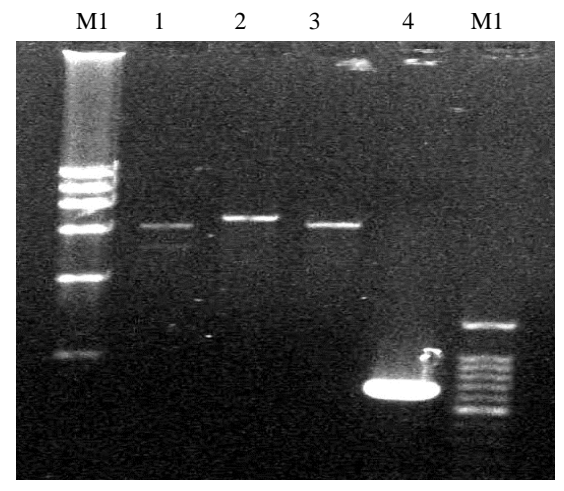

Fig. 4: Identification of double digested of PET28a. 1: Double digested products of PET28a with BamHI and XhoI (5369 bp); M: $\lambda$ Hind; DNA Marker

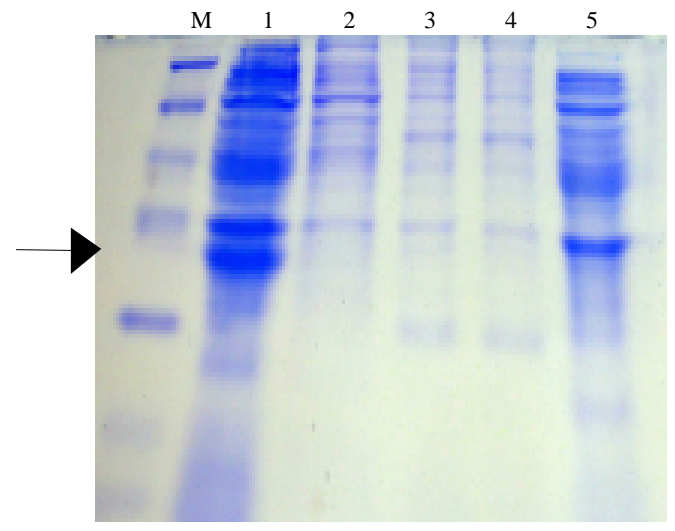

Fig. 5: Identification of recombinant plasmid M1: DL 15000 bp DNA Marker; M2: 100 bp DNA Marker; 1, 3: Double digested products of pET-VP1 with BamHI and XhoI; 2: Single digested products of pET-VP1 with XhoI $4: V P 1$ gene 


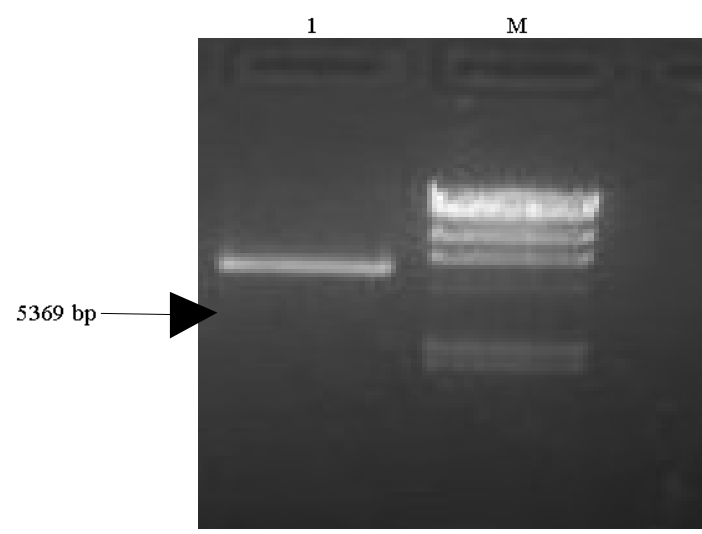

Fig. 6: SDS-PAGE analysis of expressed products; M: Prestained protein Marker; 1: The precipitation of pET-VP1 after ultrasonication; 2: The supernatant of pET-VP1 after ultrasonication 3: BL21+pET-VP1; 4: pET28a; 5: BL21

observed obvious expression product strip (Fig. 1-6). Western-blotting identification was took with swineanti-FMDV high immune serum. Obvious color reaction bands which indicated that this expression product was consistent with expection appeared in the place of expression protein.

Nucleotide sequences analysis about $V P 1$ gene: In this test, structural protein $V P 1$ genes of FMDV was amplified and nucleotides sequence analysised which indicated that researchers obtained complete VP1 genes of FMDV. According to Rice-Heasse research theory on small RNA viraceae polioviruses, strain differences between the homologie of nucleotides sequence was $<5 \%$ for genetic relationship closely. The results of sequences comparison show that the isolated strains of VP1 sequence had the highest homologie with China's Tibet separate strains O-Tibet-99 in 1999 and Japanese separation strains O-JPN-00 in 2000 reaching 96.2 and $95.9 \%$, respectively for genetic relationship closely. But the homologie of nucleotide sequence with O-ISA-8-83, O-ISA-1-74 and O-JAV-5-72 were lower, reaching 24.4 and $23.9 \%$, respectively.

Epression of VP1 genes in Escherichia coli: Taking recombinant plasmid $\mathrm{pET}-\mathrm{VP1}$ as templates amplified and obtained complete VP1 genes with specific expression primer and high-fidelity pfuDNA polymerase. PET system could express soluble protein with six histidine. The restriction enzyme sites of Enterokinase could be used to cut fusion protein which were convenient for purification and research. In this test, recombinant plasmid pET-P1 was constructed with PET system was expressed in Escherichia coli with IPTG inducing. There was no soluble protein expression in bacteria pyrolysis supernatant which indated that expression protein mainly existed with the form of inclusion body. The protein products of expression had specific reaction with swine FMDV positive serum which indated that structural protein VP1 genes of FMDV can be expressed in Escherichia coli. The molecular weight of actual expression product was the same size with the reading frame of pET-VP1 which was constructed. It indated that expression product is a kind of fusion protein. Further experiments proofed that the fusion protein exist with the form of inclusion body.

\begin{abstract}
About specificity of expression product of $V P 1$ gene: Foot-and-mouth disease virus structural protein $V P 1$ gene contained the main epitope of FMDV. Amino acid residues of VP1 protein at the 141 160 and 200 213 bits all were FMDV epitope, playing a decisive role in the research on FMD (Mateu, 1995). Suryanarayana et al. (1999) took VP1 1/2C end part of FMDV express in Escherichia coli. Product protein was detected by immunoprecipitation reaction after adsorption purified. It proofed that type specific antibodies produce. This kind of expression protein and antibodies could be used as diagnosed reagents for FMD.
\end{abstract}

About activity of expression product of $V P 1$ gene: In this test, VP1 genetic fragment was cloned by PCR and expressed in Escherichia coli. The protein products of expression could occur specificity reaction with swine FMDV positive serum. It provided technical support for expressing whole structural protein Plof FMDV and can be used to explore FMD detection methods taking expression product as a diagnostic antigens.

\section{CONCLUSION}

In this study, results showed that the structural protein VP1 gene of FMDV can express successfully in E. coli. Molecular weight of the fusion protein was $28 \mathrm{kD}$. The fusion protein can be recognized by the positive serum of swine that was infected with FMDV.

\section{REFERENCES}

Francisco, S., S. Margarita and A. Miguel, 2001. Foot-andmouth disease virus: Along known virus, but a current threat. Vet. Res., 32: 12-30. 
Kleid, D.G., 1981. Cloned viral protein vaccine for footand-mouth disease responses in cattle and swine. Science, 214: 1125-1129.

Logan, D., R. Abu-Ghazaleh and W. Blakemore, 1993. Structure of a major immunogenic site on foot-andmouth disease virus. Nature, 362: 566-568.

Mateu, M.G., 1995. Antibody recognition of picornaviruses and escape from neutralization: A structural view. Virus Res., 38: 1-24.
Suryanarayana, V.V.S., S. Viswanathan and G. Ratish, 1999. E. coli expressed proteinsasdiagnosticreagents for typing of foot-and-mouth disease virus. Arch. Virol., 144: 1701-1712.

Ward, G., E. Rieder and P.W. Mason, 1997. Plasmid DNA encoding replicating foot-and-mouth disease virus genomes induces antiviral immune responses in swine. J. Virol., 71: 7442-7447. 